\title{
BMJ Open iColon, a patient-focused mobile application for perioperative care in colorectal surgery: an observational, real-world study protocol
}

\author{
Elisa Bertocchi (D) , ${ }^{1}$ Giuliano Barugola, ${ }^{1}$ Irene Gentile, ${ }^{1}$ Teresa Zuppini, ${ }^{2}$ \\ Massimo Zamperini, ${ }^{3}$ Massimo Guerriero (D) , ${ }^{4,5}$ Renato Avesani, ${ }^{6}$ \\ Silvia Bonadiman, ${ }^{6}$ Chiara Anselmi, ${ }^{7}$ Giacomo Ruffo ${ }^{1}$
}

To cite: Bertocchi E, Barugola G, Gentile I, et al. iColon, a patientfocused mobile application for perioperative care in colorectal surgery: an observational, realworld study protocol. BMJ Open 2021;11:e045526. doi:10.1136/ bmjopen-2020-045526

- Prepublication history for this paper is available online To view these files, please visit the journal online (http://dx.doi. org/10.1136/bmjopen-2020045526).

Received 03 October 2020 Accepted 06 August 2021
Check for updates

(C) Author(s) (or their employer(s)) 2021. Re-use permitted under CC BY-NC. No commercial re-use. See rights and permissions. Published by BMJ.

For numbered affiliations see end of article.

Correspondence to

Dr Elisa Bertocchi;

elisa.bertocchi@sacrocuore.it

\section{ABSTRACT}

Introduction The enhanced recovery after surgery (ERAS) protocol provides optimised care guidelines for patients undergoing elective colorectal surgery. To ensure high compliance with active ERAS elements, patients must be educated to actively participate in the perioperative care pathway. Mobile health is a rapidly expanding area of the digital health sector that is effective in educating and engaging patients during follow-up. iColon is a mobile application designed by the Operative Unit of General Surgery of IRCCS Sacro Cuore Don Calabria Hospital of Negrar of Valpolicella, which is specifically targeted at patients undergoing elective colorectal surgery. iColon is organised into ERAS phases, and it provides real-time feedback to surgeons about a patient's adherence to perioperative active ERAS elements.

Methods and analysis We hypothesise that by providing a patient-focused mobile application, compliance with active ERAS elements could be improved.

The first coprimary objective is to build patient confidence in using the mobile application, iColon, during perioperative care. The second coprimary objective is to establish patient compliance with active ERAS elements.

Secondary objectives include examining: length of stay, 30-day readmission rate, postoperative complications and patient satisfaction of received care.

This study is a prospective observational real-world study of patients undergoing elective colorectal surgery who are following the ERAS protocol and using iColon during perioperative periods between September 2020 and December 2022.

By educating and engaging patients in the ERAS protocol, the mobile application, iColon, should stimulate patients to be more proactive in managing their healthcare by complying more closely with active ERAS elements.

Ethics and dissemination This study has been approved by the local Ethics Committee with the protocol number 29219 of 25 May 2020 . The results will be actively disseminated through peer-reviewed journals, conference presentations and various community engagement activities.
Strengths and limitations of this study

- Determine the real user confidence in a mobile application among patients undergoing elective colorectal surgery acquiring daily data from real-life patients as opposed to the selected population of a randomised clinical trial.

- Effective evaluation of patients' compliance with preoperative and postoperative active items of enhanced recovery after surgery protocol.

- Continuous data collection and analysis throughout the day thanks to the real-time feedback to the surgeons provided by the application iColon.

- Limited by being single centre based and observational in nature.

\section{INTRODUCTION}

The enhanced recovery after surgery (ERAS) protocol is an evidence-based standardised multimodal programme formulated to reduce surgical stress and optimise recovery after surgery. ${ }^{1-3}$ The ERAS represents the best care practice for patients undergoing colorectal surgery. ${ }^{1-6}$ The protocol elements, denoted as 'items', are organised into four phases. Adherence to the majority of said items has been shown to improve recovery after surgery and postoperative outcomes. ${ }^{167}$

As described by Thorn et al, ERAS items can be divided into 'active', whereby partial or full compliance by the patient is required, and 'passive', which relates to the clinician's activities and does not require the patient's input. ${ }^{8}$ For 'active' items to be effectively implemented, patient education and participation are fundamental. Fortunately, the digital health sector presents an opportunity to address them both. ${ }^{9-12}$

Mobile health is a field in which medical practices and public health are supported by mobile communication devices. It has been 
created for health services, to help ensure adequate follow-up and data collection. Some mobile health platforms can be extremely beneficial tools for patient education, stimulation, engagement and follow-up. Moreover, they can collect data about perioperative care. $^{9-13}$

The ERAS protocol for elective colorectal surgery was standardised by the multidisciplinary team of the IRCCS Sacro Cuore Don Calabria Hospital of Negrar of Valpolicella in September 2018. In our experience, typical adherence rate to active ERAS items, among patients, ranges from $40 \%$ to $85 \%$ per element. Preoperative and postoperative active items are reported to have the lowest adherence in particular. During the hospitalisation, adherence to active items is higher possibly due to the motivation and support of nurses and physiotherapists. In our hospital, the preoperative items with the lowest adherence rates were prehabilitation (55\%) and immunonutrition $(75 \%)$ and the postoperative item with the lowest adherence was active mobilisation during the day. Similar observations of which have also been reported elsewhere in the literature. ${ }^{4810}$

We hypothesise that by providing a patient-focused mobile application, named iColon, compliance with the active elements of the ERAS protocol could be improved, thus resulting in increased patient satisfaction and an improved quality of life.

\section{METHODS AND ANALYSIS \\ Objectives}

The first coprimary objective is to evaluate patient adherence to and confidence in using the mobile application, iColon, during perioperative care defined by the successful compilation of the app's daily diary section by the patient (yes/no response vs missing value).

The second coprimary objective is to evaluate patient's compliance with preoperative and postoperative active ERAS items and corresponds specifically to the yes versus no answer given for each active item.

Secondary objectives are to determine the length of stay, 30-day readmission rate, postoperative complications as according to Clavien and Dindo classification ${ }^{14}$ and patient satisfaction regarding received care.

iColon-a mobile application iColon is a customised and free of charge mobile application designed by the surgeons of the Operative Unit of General Surgery of IRCCS Sacro Cuore Don Calabria Hospital of Negrar of Valpolicella, which is specifically aimed at patients undergoing elective colorectal surgery. iColon works on smartphones with the operating systems, iOS V.11.1 (release date: 22 March 2020) and up or Android V.5.0 (release date: 19 March 2020) and up. It is organised into ERAS phases (figure 1), and contains educational sections with videos and explanatory texts. iColon sends push notifications twice daily to remind patients to record their data

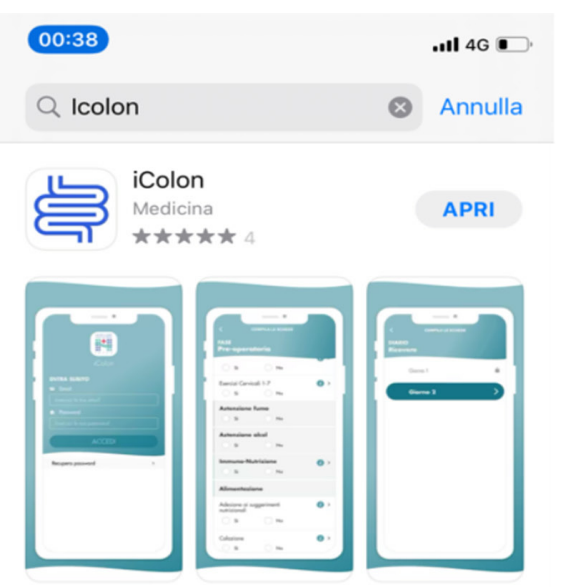

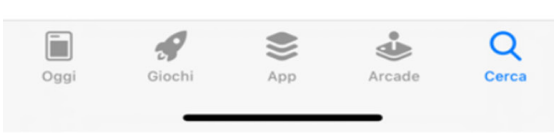

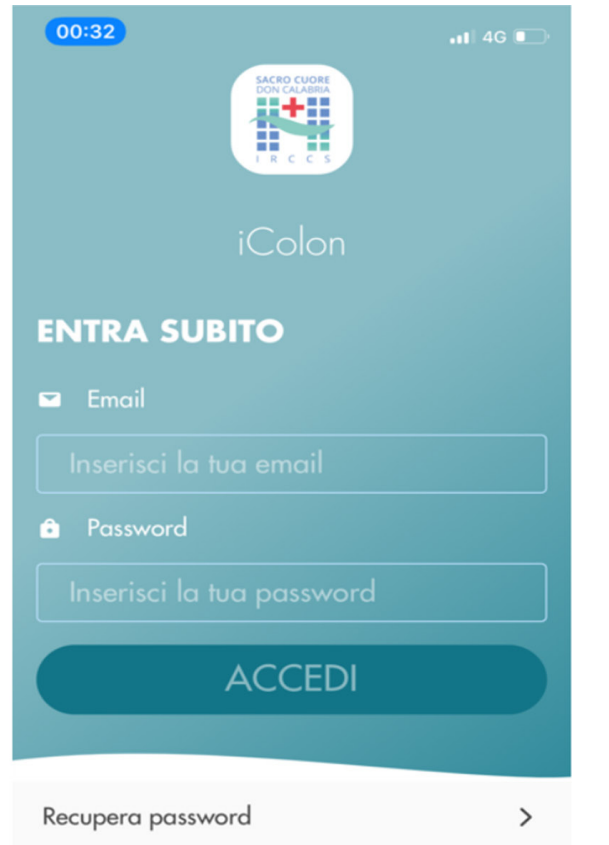

Figure 1 Screenshot of iColon mobile application showing its display on the apple store main page and the user interface for the log-in page. 
for active ERAS items. It provides real-time feedback, and surgeons can monitor a patient's perioperative progress on a dedicated web-app. During the postdischarge phase, in cases of critical issues such as fever, increased stoma output, difficult pain control, alternated bowel functions and reduced feeding, a specific alert is sent to surgeons.

During the preoperative phase, the main aims are patient prehabilitation, nutritional optimisation and effective communication between surgeon and patient. Starting 7 days prior to surgery, patients will complete a daily diary as shown in table 1 . At the end of the diary, an open field for communication between the patient and clinician is available to facilitate the resolution of any preoperative concerns.

During the hospitalisation, the principal goals after surgery are early intake of oral fluids and solids, and early mobilisation. From the day of surgery, iColon helps educate patients to reliably record any pain, nausea and vomiting, bowel movements and, for those with a stoma, it explains how to manage and quantify its output. Table 1 shows an example of a daily diary page during the hospital stay.

After discharge, patients will receive notifications to remind them to update their daily diary as shown in table 1. As in the preoperative phase, an open field for communication between patient and clinician is available.

At the end of the ERAS protocol, patients will complete a satisfaction survey about the usability of the mobile application during the perioperative care period and about their confidence in using it filling a detailed textual survey.

Patients' data will remain available to access on the webapp database for 20 days following discharge and then will be automatically downloaded and stored in the medical records of the IRCCS Sacro Cuore Don Calabria Hospital as a pdf. document.

The usability of iColon was initially tested in March 2020 by staff and again in May 2020 by patients undergoing colorectal surgery. A series of adjustments have since been made, and iColon will be continuously monitored to apply further improvements if needed. The surgical team that created it are currently working on an update to better protect surgical patients during the SARS-CoV-2 outbreak using a specific checklist before hospital admission and again after discharge to identify any individuals that may have developed symptoms, and to trace their recent contacts. ${ }^{15}$

\section{Patient recruitment}

The mobile application, iColon, is explained and offered to all patients undergoing elective colorectal surgery during the preadmission phase, at least 10 days before surgery.

Once a patient agrees to use iColon, the surgeon enters their data on the web-app, generating a personal password for patient access to the application.

The discharge date will be entered on the web-app, and a postdischarge diary will be automatically generated.

\section{Study design and duration}

This is a prospective, observational real-world study of patients undergoing elective colorectal surgery, who are following the ERAS protocol, and who will use the mobile application, iColon, during the perioperative period at IRCCS Sacro Cuore Don Calabria Hospital between September 2020 and December 2022. An interim analysis is also planned for November 2021.

The study is to be developed in accordance with the 'STrengthening the Reporting of Observational Studies in Epidemiology' checklist.

\section{Sample selection}

Inclusion criteria are:

- Age $\geq 18$ years.

- Patients in possession or cohabiting with family members in possession of a smartphone or tablet using iOS V.11.1 and up or Android V.5.0 and up operating systems.

- Patients undergoing elective colorectal surgery. Elective colorectal surgery will include all surgical colorectal procedures conducted outside of emergency or urgency settings, on patients in a stable haemodynamic condition and with a complete preoperative diagnostic workup.

- Patients following the ERAS protocol.

- Benign and malignant baseline diseases.

- Laparoscopic and laparotomic surgical techniques.

- Patients with and without stoma creation.

- Ppatients' written acceptance to be enrolled in the study.

- Patients' written consent to personal data treatment. Exclusion criteria are:

- Palliative surgery.

- Urgent and emergency surgery.

- Inability to understand the Italian language.

- Visual impairment.

- Physical or mental disabilities that limit the use of the mobile application.

Patients who decline to download iColon will receive a printed brochure of the ERAS protocol. Patients who decline to participate in the observational study will still be permitted to use iColon as per normal practice.

\section{Intervention to be measured}

Table 1 shows the active ERAS elements included in the iColon app according to the different phases of the ERAS protocol. Data relating to patient adherence in using iColon is defined as the compilation (or not) of the daily diary (yes/no response vs missing value) whereas patient compliance to the ERAS protocol is defined as yes vs no responses to each active item.

Table 2 reports demographic and clinical data to be input by the surgeon into the web-app at the point of patient recruitment. These data, with the exceptions of 'anaemia screening' and 'type of surgery planned', will be collected as predictive values for adherence in using iColon and for adherence to active ERAS items. 
Table 1 Active ERAS elements recorded in the iColon application

Preoperative phase from day 7 to day 2

Aerobic activity

Cervical exercises

Upper limb exercises

Cessation of smoking and alcohol intake

Following dietitian's nutritional advice

Immunonutrition

Preoperative phase day 1

Aerobic activity

Cervical exercises

Upper limb exercises

Cessation of smoking and alcohol intake

Following dietitian's nutritional advice Immunonutrition

Make-up removal

Depilation

Shower

Carbohydrate loading in the evening before surgery

Prophylaxis against venous thromboembolism

Day of surgery

Carbohydrate loading 2 hours before surgery

Yes/no/missing value

Postoperative phase hospitalisation

Pain evaluation according to the pain intensity scale from 0 to 10 , three times per day

Vomiting and/or nausea

Fever

Bowel movements

Stoma output reporting the type of stool

Chewing gum $15 \mathrm{~min}$ three times a day

Feeding (breakfast, lunch, dinner)

Exercises in bed

Breathing exercises

Eating out of bed

Out of bed for at least 4-6hours

Postoperative phase, postdischarge diary, from day one after discharge to day 5 after discharge

\begin{tabular}{ll} 
Pain evaluation according to the pain intensity scale from 0 to 10 & From 0 to 10 points \\
Vomiting and/or nausea & Yes/no/missing value \\
Fever & Yes/no/missing value \\
Bowel movements & Yes/no/missing value \\
Stoma output reporting the type of stool & Free field \\
Feeding (breakfast, lunch, dinner) & Yes/no/missing value \\
Out of bed for at least 4-6hours & Yes/no/missing value \\
\hline Prophylaxis against venous thromboembolism & Yes/no/missing value
\end{tabular}

ERAS, enhanced recovery after surgery.

Table 3 lists the surgical details and postoperative outcomes to be entered into the web-app by the surgeon after patient discharge. These data will be analysed in order to define the correlation between the adherence to active ERAS items and surgical and postoperative outcomes. 
Table 2 Demographic and clinical data of patients registered on the iColon web-app platform

\section{Demographic and clinical data}

\begin{tabular}{ll}
\hline Age & (18-99years) \\
Sex & Male \\
Female & 1: A normal healthy patient \\
2: A patient with mild systemic \\
disease \\
3: A patient with severe \\
systemic disease \\
4: A patient with severe \\
systemic disease that is a \\
constant threat to life
\end{tabular}

ASA, American Society of Anesthesiologists.

\section{Sample size calculation}

Considering the number of cases typically treated at the hospital, we estimate enrolling approximately 120 subjects per year, which would be about 270 consecutive patients between September 2020 and December 2022.

\section{Statistical analysis: data analysis plan}

General statistical analysis strategy

Demographic and clinical data will be summarised using descriptive statistics, measures of variability and precision, and plots. Statistical tests will be used based on the type of variables, tests assumptions and sample dimension. All parameters will be reported with $95 \%$ CIs. Statistical models and estimations will be adjusted for covariates if necessary. Information criteria and likelihood ratio methods will be used to compare candidate models.

\section{Analysis of primary objectives}

Concerning adherence to the use of iColon, the ratio between the number of patients using iColon for at least $75 \%$ of responses (yes/no response vs missing value), and the total number of patients, will be calculated. A multivariate logistic model will be used to analyse adherence
Table 3 Surgical details and postoperative outcomes of patients registered on the iColon web-app platform

\section{Surgical details and postoperative outcomes}

\begin{tabular}{|c|c|}
\hline Surgical technique & $\begin{array}{l}\text { Laparoscopy } \\
\text { Laparotomy }\end{array}$ \\
\hline Length of surgery & In minutes \\
\hline Abdominal drain & Yes/no \\
\hline Presence of stoma & Yes/no \\
\hline $\begin{array}{l}\text { Urinary catheter removed on } \\
\text { the first post-operative day }\end{array}$ & Yes/no \\
\hline Anastomotic leak & Yes/no \\
\hline $\begin{array}{l}\text { Postoperative complication } \\
\text { as according to Clavien and } \\
\text { Dindo classification }\end{array}$ & From grade 1 to grade 5 \\
\hline $\begin{array}{l}\text { Postoperative blood } \\
\text { transfusion }\end{array}$ & Yes/no \\
\hline No of days of hospitalisation & $\begin{array}{l}\text { Automatically generated } \\
\text { after entering the discharge } \\
\text { date }\end{array}$ \\
\hline $\begin{array}{l}\text { Readmission within thirty days } \\
\text { after discharge }\end{array}$ & Yes/no \\
\hline $\begin{array}{l}\text { Thirty-day postoperative } \\
\text { mortality }\end{array}$ & Yes/no \\
\hline
\end{tabular}

with a set of predictor variables and confounders collected in iColon.

The predictor variables and the confounders that will be considered are age, sex, type of disease, presence of inflammatory bowel disease (IBD), postoperative complication according to Clavien and Dindo classification for the postoperative phases, anastomotic leak, number of days of hospitalisation.

Concerning confidence in the usability of iColon, the ratio between the number of patients submitting positive feedback (defined as 4 or 5 points) in the satisfaction survey, and the total number of patients, will be calculated. A multivariate logistic model will be used to analyse confidence (positive feedback defined as 4 or 5 vs not positive feedback defined as 1 or 2 or 3 ) with a set of predictor variables and confounders collected in iColon. The predictor variables and the confounders that will be considered are age, sex, type of disease, presence of IBD, postoperative complications according to Clavien and Dindo classification for the postoperative phases, anastomotic leak for the postoperative phases, number of days of hospitalisation for the postoperative phases.

Concerning the compliance to active ERAS items, the ratio between the number of patients following at least $75 \%$ of preoperative and postoperative active ERAS items and the total number of patients will be calculated. A multivariate logistic model will be used to analyse preoperative and postoperative active ERAS items (yes vs no) with a set of predictor variables and confounders collected in iColon. The predictor variables and the confounders that will be considered are age, sex, type of disease, American 
Society of Anesthesiologists (ASA) status, presence of IBD, type of surgery planned for all phases of perioperative care. For the postoperative phases (hospitalisation-postdischarge) the following predictors will be considered: surgical technique, presence of stoma, anastomotic leak, postoperative complications as according to Clavien and Dindo classification, number of days of hospitalisation, readmission within thirty days after discharge.

\section{Analysis of secondary objectives}

Descriptive statistics and frequency distribution will be performed to describe length of stay, readmission rate, postoperative complications and patient satisfaction of care.

\section{Ethics and dissemination policy}

The data will be owned by the Operative Unit of General Surgery of the IRCCS Sacro Cuore-Don Calabria Hospital of Negrar of Valpolicella (VR).

The data collected in paper form will be entered into a dedicated database. Access to data will be allowed only to the authorised personnel directly involved in the study (principal investigator, and collaborators).

The data will be kept confidential and will be processed in full compliance with Legislative Decree 196/03 and GDPR 679/2016 (protection of people and other subjects regarding the processing of personal data), under which all participants will be asked to sign informed consent and consent to the processing of personal data.

Study results will be used for the preparation of scientific papers and presentation in conferences.

The latest revision of the Helsinki declaration as well as the Oviedo declaration are the basis for the ethical conduct of the study.

The local Ethics Committee have examined all the documentation in order to safeguard the subjects' rights and confidentiality and have approved the study protocol (protocol number 29219-25 May 2020).

The protocol and all documentation relating to this study, and any revisions to these documents, will be used only with the authorisation of the local ethics committee. All essential clinical documents will be kept demonstrating the validity of the study and the integrity of the data collected.

\section{Patient and public involvement}

No patients were involved in the development of the research and in the outcome measures.

No patients were involved in the design of this study.

No patients were involved in the recruitment to and conduct of the study.

The results of this study will be published in a scientific journal.

\section{DISCUSSION}

ERAS guidelines suggest that patients themselves play the main role in preoperative optimisation and postoperative recovery. ${ }^{1-4}$ For these reasons, their education and participation in the pathway of perioperative care are key in obtaining positive outcomes. ${ }^{13510}$ iColon is designed to help patients undergoing colorectal surgery by providing customised care.

Other studies have evaluated or are evaluating the role of mobile health as a tool to prompt, educate and monitor patients during their perioperative care for colorectal, bariatric and spine surgery. ${ }^{9-13}$ 16-18 These studies are generally randomised clinical trials (RCTs) involving selected populations of patients. ${ }^{9} 111216-19$ In Italy, no other mobile tool like iColon is known to be in use. Another similar mobile application, in use in Canada, is reported by Mata et al in a recent paper. ${ }^{19}$ The Amsterdam University Medical Centres (UMCs) are testing a similar mobile application but with different inclusion and exclusion criteria. ${ }^{9}$ The main differences between iColon and the majority of other mobile health applications available is that iColon offers mobile support during the entire perioperative period. It is all at once an educational tool with videos and explanatory texts, a motivational tool with push notifications that remind patients to follow ERAS items and to record data, and a follow-up tool with a daily diary that relays immediate feedback to the surgeons on the webapp. In our study protocol, we intend to evaluate not only the effect of iColon on adherence to active ERAS items, but also to determine user confidence in a mobile application among patients undergoing elective colorectal surgery in real-life medical practice. Real-world evidence studies, such as this, allow us to acquire daily data from real-life patients. The design of a RCT represents the next step based on the results of this study in order to validate the scientific evidence. As such, we hope to gain an alternative perspective of user confidence and adherence to active ERAS items.

We hypothesise that iColon is a valid and useful tool for patients undergoing elective colorectal surgery. By encouraging compliance with active ERAS items, iColon should stimulate patients to be more mindful of their own health and to comply more with active ERAS items.

\section{Ethics approval statement}

This study involves human participants and was approved by an Ethics Committee. Ethics name ID: Comitato Etico delle Province di Verona e Rovigo. Reference number of ethical approval: protocol number 29219 of 25 May 2020.

\section{Author affiliations}

${ }^{1}$ General Surgery Unit, IRCCS Sacro Cuore Don Calabria Hospital, Negrar di Valpolicella, Verona, Italy

${ }^{2}$ Hospital Pharmacy, IRCCS Sacro Cuore Don Calabria Hospital, Negrar di Valpolicella, Verona, Italy

${ }^{3}$ Department of Anaesthesia, Intensive Care and Pain Therapy, IRCCS Sacro Cuore Don Calabria Hospital, Negrar di Valpolicella, Verona, Italy

${ }^{4}$ School of Medicine and Surgery, University of Verona, Verona, Italy

${ }^{5}$ Clinical Research Unit, IRCCS Sacro Cuore Don Calabria Hospital, Negrar di Valpolicella, Verona, Italy

${ }^{6}$ Rehabilitation Department, IRCCS Sacro Cuore Don Calabria Hospital, Negrar di Valpolicella, Verona, Italy

${ }^{7}$ Dietetic Service, IRCCS Sacro Cuore Don Calabria Hospital, Negrar di Valpolicella, Verona, Italy 
Acknowledgements The authors thank Elinor Julie Rae Anderson, medical writer freelance, for her linguistic revision of the manuscript.

Contributors EB: Design of the mobile application's structure, development and the management of iColon. Design of the study protocol. Data collection. Drafting of the manuscript of the study protocol. GB: Design of the mobile application's structure, development and the management of iColon. Design of the study protocol. IG: Design of the mobile application's structure, development and the management of iColon. TZ: Contributtion to the study design and to the creation of the educational sections of the application iColon. MZ: Contributtion to the study design and to the creation of the educational sections of the application iColon. RA: Contributtion to the study design and to the creation of the educational sections of the application iColon. SB: Contributtion to the study design and to the creation of the educational sections of the application iColon. CA: Contributtion to the study design and to the creation of the educational sections of the application iColon. MG: statistical analysis and data management. GR: Direction of all study components. Design of the mobile application's structure, development and the management of iColon. All authors read and approved the final version of the manuscript.

Funding The present publication was supported by Johnson \& Johnson Medical S.p.A, who owns the intellectual and industrial property rights of iColon mobile application.

Competing interests None declared.

Patient and public involvement Patients and/or the public were not involved in the design, or conduct, or reporting, or dissemination plans of this research.

Patient consent for publication Not applicable.

Provenance and peer review Not commissioned; externally peer reviewed.

Open access This is an open access article distributed in accordance with the Creative Commons Attribution Non Commercial (CC BY-NC 4.0) license, which permits others to distribute, remix, adapt, build upon this work non-commercially, and license their derivative works on different terms, provided the original work is properly cited, appropriate credit is given, any changes made indicated, and the use is non-commercial. See: http://creativecommons.org/licenses/by-nc/4.0/.

\section{ORCID iDs}

Elisa Bertocchi http://orcid.org/0000-0002-8671-6493

Massimo Guerriero http://orcid.org/0000-0003-1310-539X

\section{REFERENCES}

1 Gustafsson UO, Scott MJ, Hubner M, et al. Guidelines for perioperative care in elective colorectal surgery: enhanced recovery after surgery (ERAS ${ }^{\circledR}$ ) society recommendations: 2018. World J Surg 2019;43:659-95.

2 Fearon $\mathrm{KCH}$, Ljungqvist $\mathrm{O}$, Von Meyenfeldt $\mathrm{M}$, et al. Enhanced recovery after surgery: a consensus review of clinical care for patients undergoing colonic resection. Clin Nutr 2005;24:466-77.

3 Varadhan KK, Neal KR, Dejong CHC, et al. The enhanced recovery after surgery (ERAS) pathway for patients undergoing major elective open colorectal surgery: a meta-analysis of randomized controlled trials. Clin Nutr 2010;29:434-40.

4 Vlug MS, Wind J, Hollmann MW, et al. Laparoscopy in combination with fast track multimodal management is the best perioperative strategy in patients undergoing colonic surgery: a randomized clinical trial (LAFA-study). Ann Surg 2011;254:868-75.

5 Spanjersberg WR, Reurings J, Keus F, et al. Fast track surgery versus conventional recovery strategies for colorectal surgery. Cochrane Database Syst Rev 2011;16:CD007635.

6 Ljungqvist O, Scott M, Fearon KC. Enhanced recovery after surgery: a review. JAMA Surg 2017;152:292-8.

7 Pecorelli N, Hershorn O, Baldini G, et al. Impact of adherence to care pathway interventions on recovery following bowel resection within an established enhanced recovery program. Surg Endosc 2017:31:1760-71.

8 Thorn CC, White I, Burch J, et al. Active and passive compliance in an enhanced recovery programme. Int $J$ Colorectal Dis 2016;31:1329-39.

9 Rauwerdink A, Jansen M, de Borgie CAJM, et al. Improving enhanced recovery after surgery (ERAS): eras APPtimize study protocol, a randomized controlled trial investigating the effect of a patient-centred mobile application on patient participation in colorectal surgery. BMC Surg 2019;19:125.

10 Pecorelli N, Fiore JF, Kaneva P, et al. An app for patient education and self-audit within an enhanced recovery program for bowel surgery: a pilot study assessing validity and usability. Surg Endosc 2018;32:2263-73.

11 Bednarski BK, Nickerson TP, You YN, et al. Randomized clinical trial of accelerated enhanced recovery after minimally invasive colorectal cancer surgery (RecoverMI trial). Br J Surg 2019;106:1311-8.

12 Kim B-Y, Park K-J, Ryoo S-B. Effects of a mobile educational program for colorectal cancer patients undergoing the enhanced recovery after surgery. Open Nurs J 2018;12:142-54.

13 Pecorelli N, Hershorn O, Baldini G, et al. Impact of adherence to care pathway interventions on recovery following bowel resection within an established enhanced recovery program. Surg Endosc 2017;31:1760-71.

14 Dindo D, Demartines N, Clavien P-A. Classification of surgical complications: a new proposal with evaluation in a cohort of 6336 patients and results of a survey. Ann Surg 2004;240:205-13.

15 Barugola G, Bertocchi E, Ruffo G. Stay safe stay connected: surgical mobile app at the time of Covid-19 outbreak. Int $J$ Colorectal Dis 2020;35:1781-2.

16 Glauser G, Ali ZS, Gardiner D, et al. Assessing the utility of an loS application in the perioperative care of spine surgery patients: the NeuroPath pilot study. Mhealth 2019;5:40.

17 Mangieri CW, Johnson RJ, Sweeney LB, et al. Mobile health applications enhance weight loss efficacy following bariatric surgery. Obes Res Clin Pract 2019;13:176-9.

18 Eisenhauer CM, Brito FA, Yoder AM, et al. Mobile technology intervention for weight loss in rural men: protocol for a pilot pragmatic randomised controlled trial. BMJ Open 2020;10:e035089.

19 Mata J, Pecorelli N, Kaneva P, et al. A mobile device application (app) to improve adherence to an enhanced recovery program for colorectal surgery: a randomized controlled trial. Surg Endosc 2020;34:742-51. 